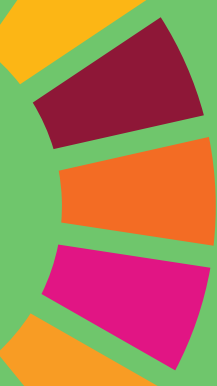

\title{
EL FONDO DE ADAPTACIÓN COMO INSTRUMENTO DE ACCESO A LA FINANCIACIÓN CLIMÁTICA PARA LAS COMUNIDADES LOCALES
}

\section{THE ADAPTATION FUND AS AN INSTRUMENT OF ACCESS TO CLIMATE FINANCE FOR LOCAL COMMUNITIES}

\author{
Alicia Tortosa García \\ Institución donde trabajo: Fundación ETEA - Instituto de \\ Desarrollo de la Universidad Loyola Andalucía \\ ali88tg@gmail.com
}

Fecha recepción artículo: 08/03/2021 • Fecha aprobación artículo: 03/05/2021

\section{RESUMEN}

El Grupo Intergubernamental de Expertos sobre el Cambio Climático (IPCC) ha demostrado que los esfuerzos orientados a la mitigación son insuficientes para frenar dicho fenómeno. Esto se produce debido a que los/as expertos/as han demostrado que muchos de sus efectos ya son irreversibles para muchas poblaciones, por lo que la adaptación se ha convertido en una acción esencial en las estrategias diseñadas para enfrentar el cambio climático. Sin embargo, al ser la adaptación gran dependiente del contexto donde se produce, la mayoría de su eficacia depende de la incorporación de las comunidades locales en los procesos de adaptación. El Fondo de Adaptación, uno de los instrumentos financieros de la Convención Marco de Naciones Unidas sobre el Cambio Climático (CMUNCC), contiene una serie de características que a priori indican que facilitan el acceso a la financiación climática a las comunidades. Así, el presente artículo analizará cómo dichas características benefician o no la mayor canalización de financiación climática hacia las comunidades locales a través de las organizaciones locales y las Entidades Implementadoras Nacionales (NIEs por sus siglas en inglés). Para evidenciar lo anterior, se mostrará brevemente un estudio de caso.

Palabras clave: Adaptación, Financiación climática, Cambio climático, Comunidad, Acceso directo, Gobernanza. 


\section{ABSTRACT}

The Intergovernmental Group of Experts on Climate Change(IPCC) has shown that efforts towards mitigation are insufficient to stop this phenomenon. This occurs because experts have shown that many of its effects are already irreversible for many populations, so adaptation has become an essential action in strategies designed to face climate change. However, since adaptation is highly dependent on the context where it happens, its effectiveness will depend on the incorporation of local communities in adaptation processes. The Adaptation Fund, one of the financial instruments of the United Nations Framework Convention on Climate Change (UNFCCC), contains a series of characteristics that a priori indicate that they facilitate access to climate finance for communities. Thus, this article will analyze how these characteristics benefit or not the greater channeling of climate finance to local communities through local organizations and National Implementing Entities (NIEs). To demonstrate the above, a case study will be briefly shown.

Key words: Adaptation, Climate Finance, Climate Change, Community, Direct Access, Governance

Alicia Tortosa García. Graduada en Relaciones Internacionales por la UCMymáster en Cooperación Internacional por la UCM y la UPM. Ha trabajado en distintas instituciones internacionales, siendo la más reciente el Instituto Interamericano de Cooperación para la Agricultura en Costa Rica. Actualmente es Gestora de proyectos en la Fundación ETEA- Instituto de Desarrollo de la Universidad Loyola Andalucía.

\section{INTRODUCCIÓN}

El cambio climático no constituye un fenómeno nuevo. Documentos como el Informe sobre la Economía del Cambio Climático, conocido como el Informe Stern (2006) o el IV Informe del IPCC (IPCC, 2007), evidencian como los seres humanos tienen una responsabilidad de hasta un $90 \%$ sobre el cambio climático y como la inacción ante dicha problemática puede causar aún mayor daño. De esta manera, compromisos como el Acuerdo de París o la Agenda 2030 han recogido la necesidad de actuación ante la emergencia climática, poniendo el énfasis en una respuesta conjunta y coordinada de toda la comunidad internacional ante el gran reto que supone este fenómeno.

El cambio climático tiene efectos diferenciados según las condiciones políticas, económicas y sociales de los lugares donde se produce (CEPAL, 2019). Organismos como el IPCC han estudiado como los efectos del cambio climático aumentan y/o profundizan aun más las desigualdades existentes en los países en desarrollo. Esto se produce en gran medida por la dependencia que tienen dichos países de sectores económicos sensibles al clima (IPCC, 2014). Así, el Índice de Riesgo Climático Global señala que "de los diez países más afectados por fenómenos meteorológicos extremos ocurridos entre 1998 y 2017, ocho de ellos se produjeron en países en desarrollo de ingresos bajos o medio-bajos" (OXFAM, 2019). Concretamente, las comunidades locales ${ }^{1}$ de los países en desarrollo son las áreas más vulnerables a los efectos del cambio climático (Fernández, 2007).

\footnotetext{
${ }^{1}$ En el presente artículo se entenderá por comunidad local como un grupo de personas que viven en zonas rurales, que comparten un conjunto de características culturales y sociales y conviven bajo las mismas reglas.
} 
Ante este contexto, la financiación climática se ha convertido en una de las principales herramientas para trabajar en la mitigación ${ }^{2}$ y adaptación ${ }^{3}$ de los países en desarrollo frente al cambio climático. La CMNUCC, creada en 1992, estableció una serie de mecanismos financieros para responder a la deuda ecológica ${ }^{4}$ que tienen los países desarrollados frente a los países en desarrollo. Esto ha llevado a la conformación de un gran entramado financiero y una gobernanza climática de gran relevancia dentro de las agendas políticas de los países (NNUU, 1992).

El Fondo de Adaptación (FA), uno de los instrumentos creados bajo la CMNUCC, destina su financiamiento climático hacia la adaptación al cambio climático. Sin embargo, la adaptación a menudo ha ocupado un lugar secundario en el estudio realizado por parte de la academia y en su tratamiento dentro de los grandes acuerdos climáticos. No obstante, constituye un tema de gran relevancia debido a que muchos de los impactos que se han producido y se producirán con motivo del calentamiento global no se podrán cambiar, por lo que la adaptación será esencial para reducir la vulnerabilidad de las poblaciones, en especial de las comunidades locales.

El presente artículo tratará de estudiar y reflexionar sobre como el Fondo de Adaptación destina parte de esa financiación climática hacia las comunidades locales de los países en desarrollo, las cuales son consideradas los lugares más vulnerables al cambio climático. Para ello, se estudiarán las características del propio Fondo de Adaptación y se presentará un ejemplo de una entidad (representante de las comunidades locales del país) que ha accedido a la financiación del FA.

\section{FINANCIACIÓN PARA LA ADAPTACIÓN AL CAMBIO CLIMÁTICO}

Para entender el financiamiento destinado a la adaptación al cambio climático es necesario realizar una breve aproximación general. La financiación climática se estableció como uno de los instrumentos principales de la CMNUCC, celebrada en 1992 (NNUU, 1992). Pese a ser un tema de gran relevancia para la consecución de los distintos compromisos internacionales, no ha existido un acuerdo de todas las Partes en cuanto a los criterios a considerar para articular una definición de financiamiento climático. No obstante, en este artículo se utilizará la definición que realizan Cabral y Bowling (2014:16) donde definen financiamiento climático como

"el proceso que involucra todos los recursos, públicos y privados, desde las promesas de canalización de fondos financieros nuevos y adicionales hasta su desembolso, monitoreo, reportey verificación, provenientes de los países del Anexo II de la Convención y destinados a los países no Anexo I (..)"

Gran parte de la complejidad del estudio de la financiación climática radica en la fragmentación de los fondos, existiendo numerosas fuentes que en ocasiones conlleva a la duplicidad de esfuerzos de financiamiento (Cabral y Bowling, 2014:75). Aunque actualmente es reconocida la necesidad de la financiación tanto para la mitigación como para la adaptación, esta siempre ha estado relegada a un segundo plano frente a

\footnotetext{
${ }^{2}$ La mitigación al cambio climático es entendida como aquella actividad dedica a disminuir la cantidad de gases de efecto invernadero en la atmósfera, al mismo tiempo que contribuye a la mejora de los sumideros (CMNUCC, 2020b).

${ }^{3}$ La adaptación al cambio climático es aquella actividad que está dedica a realizar ajustes en os ajustes en los sistemas ecológicos, sociales o económicos en respuesta a los efectos provocados o previstos por el cambio climático (CMNUCC, 2020a).

${ }^{4}$ La deuda ecológica se refiere al alto consumo de recursos naturales y producción de desechos de los países industrializados del Norte frente a los países del Sur global, lo cual ha producido efectos negativos en el medio ambiente que han repercutido de una manera más grave en estos últimos (Lago y Urkidi, n.d).
} 
la mitigación, lo que ha limitado en cierta medida su estudio teórico (Schipper, 2004:50). Tanto es así que, según un informe realizado por el Programa de las Naciones Unidas para el Medio Ambiente (PNUMA) en 2016 sobre la brecha de la adaptación, se estimaba que los recursos necesarios para garantizar una adaptación adecuada en los países en desarrollo debería ser para 2030 entre 6 y 13 veces mayores que los de ese momento (Grimm et al., 2018).

A lo largo de los años, autores tales como Burton (1994), Sands (1992), Vellinga (2003) y Pilifosova (200), han argumentado que se ha dado un mayor flujo de financiación climática hacia la mitigación debido a las siguientes razones (Schipper, 2004:51):

- En primer lugar, la adaptación se consideró una opción derrotista, ya que no se quería aceptar que el cambio climático estaba ocurriendo y debido a ello tomar acciones para combatirlo.

- En segundo lugar, hablar de adaptación conllevaba implícitamente aceptar la falta de compromiso de ciertos países en la limitación de emisiones GEl.

- Y, tercero, tratar la adaptación significaba tratar sobre la responsabilidad de quién causaba o había contribuido en mayor medida a el cambio climático. Este era un tema que los países en desarrollo aún no se atrevían a tratar puesto que temían que los compromisos de adaptación eclipsaran los compromisos que se estaban logrando sobre emisiones de GEI.

Otras de las razones que se ha mantenido para destinar más financiamiento climático hacia la mitigación que a la adaptación está respaldado por el pensamiento de que financiar acciones de mitigación producirá, en términos de largo plazo, una reducción en los costes destinados a la adaptación (Buchner et al., 2011 en Ruíz-Gauma, et al, 2020). Sin embargo, y como se señalaba anteriormente, la financiación destinada a la adaptación al cambio climático es necesaria, principalmente, porque aunque se logre reducir las emisiones que causan el cambio climático, muchos de sus efectos ya son irreversibles (MITECO, 2020).

Al mismo tiempo, la adaptación al cambio climático, además de tener un tardío desarrollo teórico, contó con un enfoque limitado. Es decir, el cambio climático se abordó solo desde el campo de las ciencias naturales, dejando fuera un enfoque social y, por tanto, reduciendo la complejidad del problema. Desde la disciplinas de las ciencias sociales se ha demostrado que las condiciones climáticas y ambientales no causan impactos en los países en desarrollo de una manera independiente, sino que existen una serie de factores socioeconómicos y/o culturales que contribuyen a dichos impactos (Bodley, 2001, en Schipper, 2004). Así, la adaptación viene a poner en valor y recoger la necesidad de incluir el estudio de variables socioeconómicas, políticas y culturales, las cuales son dependientes del contexto, y acentúan la vulnerabilidad de las poblaciones ante al cambio climático (Adger, 2008).

Schipper (2004) sostiene la necesidad de la incorporación de local y de las organizaciones de la sociedad civil para que el proceso de adaptación sea un proceso efectivo, lo que es conocido como el Enfoque Basado en la Comunidad. Este enfoque se refiere a la adaptación de abajo arriba, donde las propuestas y las necesidades de financiación para la adaptación son establecidas por la comunidad (Ornsaran, et al, 2020). La adaptación, tal y como tratan los autores Yamin, Rahman y Huq (2005), la determinan las necesidades locales y, por tanto, al ser altamente específica del contexto, tiene como base las estructuras y situaciones locales. Las comunidades deben ocupar un lugar central en los procesos de adaptación y, para ello, se debe proporcionar apoyo institucional que ayude a mejorar sus capacidades de adaptación a largo plazo (Yamin et al, 2005:1). 


\subsection{FINANCIAMIENTO PARA LA ADAPTACIÓN AL CAMBIO CLIMÁTICO EN LAS NEGOCIACIONES CLIMÁTICAS INTERNACIONALES}

Ante la preocupación sobre las consecuencias del cambio climático y la necesidad de actuación de la comunidad internacional, la Asamblea General de las Naciones Unidas aprobó en la Resolución 45/212queestablecía, a través del Comité Intergubernamental de Negociación, la Convención Marco sobre Cambio Climático (CMNUCC). Así, fruto de dicha resolución, en 1992 se adoptó la CMNUCC, cuya firma fue abierta en la Conferencia de las Naciones Unidas para el Medio Ambiente y el Desarrollo, también conocida como la "Cumbre de la Tierra". La CMNUCC se creó para estabilizar la concentración de gases de efecto invernadero (GEI) y así frenar las alteraciones en la atmósfera. Para ello, se establecieron ciertas obligaciones comunes a todas las Partes y otras específicas que recaían sobre los países del Anexo I (países industrializados y economías en transición) (Urrutia, 2010). Concretamente en el artículo 4.3, la CMNUCC estableció que los países desarrollados que conforman el Anexo I y, que habían suscrito el Anexo II, se comprometían a entregar recursos financieros nuevos y adicionales para ayudar a las Partes (países en desarrollo, también llamados no Anexo I) (Ibárcena y Scheelke, 2003).

La mitigación siempre se tomó como la solución definitiva al cambio climático y no sería hasta 2001, con la celebración de la séptima Conferencia de Naciones Unidas sobre el Cambio Climático (COP7), donde se adoptarían los Acuerdos de Marrakech. Allí se estableció el primer gran paquete sobre adaptación y creó el Fondo de Adaptación. No obstante, sería en 2007, con la celebración de la COP13 en Bali, donde la adaptación para el cambio climático se convertiría en una demanda central para los países en desarrollo (Khan et al, 2019). Ciplet et al. (2013), señalan dos factores relacionados con el porque del cambio en el foco hacia la adaptación. En primer lugar, se desarrolló más profundamente el marco cognitivo de justicia climática ${ }^{5}$, de manera que se vinculó la emisiones de GEl ${ }^{6}$ con el aumento de las catástrofes naturales ocurridas en los países del Sur. Y, en segundo lugar, después de la aprobación del Protocolo de Kioto, se crearía evidencia de que los países del Norte no estaban tomando las medidas necesarias para aumentar la emisión de GEl, ya que no se estaban logrando las metas (Ciplet et al., 2013).

En 2015 en la COP21 se firma el Acuerdo de París, donde se señala específicamente que el financiamiento climático debe ser uno de los pilares esenciales en la lucha contra el cambio climático. Los gobiernos trabajaron en definir una hoja de ruta para el aumento de la financiación climática, pero decidieron prorrogar hasta 2025 la meta de los 100000 millones acordada en la COP2, y, por primera vez, se muestra la necesidad de la participación de "otras partes" a prestar, de manera voluntaria, financiamiento (Gerenda, 2015). En el Acuerdo se señala un objetivo global en cuanto a adaptación, reconociendo su dimensión y vinculando las necesidades de adaptación con el nivel de mitigación (art. 9.4) (Khan et al, 2019). Sin embargo, el Acuerdo no fue muy específico en cuanto a herramientas y mecanismos necesarios para que dicha financiación llegará a los más vulnerables (Mostafa, et al., 2016).

No obstante, estos esfuerzos siguen siendo insuficientes, ya que sigue existiendo, como señalaba el PNUMA, una gran brecha de financiamiento para la adaptación al cambio climático (PNUMA, 2018). En la última COP25 celebrada en Madrid, la cual estuvo centrada en generar un acuerdo sobre las directrices para el mercado de carbono, los distintos actores no llegaron a un acuerdo. Este acuerdo era necesario, entre otros, para aprovechar el potencial del sector privado y generar financiación para la adaptación. Además, se dejó evidencia que los países desarrollados no habían cumplido sus compromisos con los países en desarrollo en materia de financiación, pues la meta de la consecución de los 100 mil millones de dólares está lejos de cumplirse (Gutiérrez, 2019).

\footnotetext{
5 Justicia climática es un concepto surgido a mediados de los años 80 donde se reconoce que el cambio climático es también un problema ético y político provocado en gran medida por el sistema económico actual y por los países más desarrollados, cuyos peores efectos sufren los países del Sur Global (Pardo y Ortega, 2018).

${ }^{6}$ Los GEI son aquellos que se cuentan ante la CMNUUCC y el Protocolo de Kioto e incluyen: Dióxido de Carbono, Metano, Perfluorocarbos, Hidrofluorocarbonos, Óxido nitroso y Hexafloruro de Azufre (Cabral y Bowling, 2014: 16).
} 


\section{EL FONDO DE ADAPTACIÓN}

El Fondo de Adaptación (FA en adelante) constituye uno de los fondos climáticos creados bajo la CMNUCC. Se estableció en virtud de una decisión de la Conferencia de las Partes (COP), en la COP7 celebrada en Marrakech, Marruecos, en 2001. Este instrumento financiero empezó su construcción en 2005 y no sería hasta 2010 cuando comenzaría a operar (Cabral y Bowling, 2015:45).

El FA es uno de los únicos fondos del mundo dedicado específicamente a la adaptación al cambio climático. Concretamente, está orientado a ofrecer financiación a las comunidades más vulnerables, ya que son las que más se ven afectadas por los efectos del cambio climático. El FA adopta un enfoque constructivista, el cual señala que existen factores socioeconómicos y culturales que hacen a las poblaciones más vulnerables a los efectos del calentamiento global (Lampis, 2013). Al mismo tiempo, el FA trata de aumentar la resiliencia de estas personas a través de un enfoque en las comunidades más vulnerables. Dicho enfoque concuerda con lo sostenido por autores como Schipper (2004) y Adger (2008), quienes mencionan la necesidad de la incorporación de los niveles locales en los procesos de adaptación debido a la relevancia del contexto para las acciones de adaptación para el cambio climático. Fenton y col (2014) señalan tres razones principales para explicar el éxito que ha tenido en su funcionamiento el FA (Fenton et al., 2014):

1. El mandato por el cual fue constituido a raíz del Protocolo de Kioto, establecía su constitución para centrarse en las comunidades más vulnerables al cambio climático.

2. Dentro de los requisitos que establece para aprobar fondos a las Entidades Implementadoras Nacionales (NIEs por sus siglas en inglés), se encuentra la necesidad de que dichos fondos vayan destinados a las comunidades más vulnerables.

3. La modalidad de acceso directo ofrece un alcance mayor para que las organizaciones locales planifiquen y administren proyectos financiados por el FA.

A continuación se analizarán dos de sus características más relevantes: el modelo de gobernanza y la modalidad de acceso directo del FA. Estas ayudarán a entender si los principales mecanismos y procesos establecidos por el FA facilitan una mayor canalización de los fondos del FA hacia las comunidades locales.

\subsection{EL MODELO DE GOBERNANZA DEL FONDO DE ADAPTACIÓN}

La gobernanza es una de las características del FA que le hace especial dentro de la arquitectura climática internacional. Cuando se sentaron las bases de la articulación de la gobernanza del FA se debatió sobre quién debería supervisar el mismo. Así, mientras los países desarrollados apostaban como órgano rector del FA al Fondo para el Medio Ambiente Mundial (FMAM), institución establecida en 1991 por el Banco Mundial, los países en desarrollo presionaron para que la supervisión del Fondo recayera en una institución bajo la COP, de acuerdo con el artículo 11 del Protocolo de Kioto. Los países en desarrollo se opusieron principalmente al FMAM porque estos tenían menor representación en dicho organismo y, además, suponía un mayor poder de decisión para los países desarrollados, ya que los principales donantes tienen poder de veto en el Banco Mundial (Ciplet, et al. 2013).

Finalmente, el problema fue resuelto en la COP12 de Nairobi en 2006 con la adopción de la llamada Decisión del Fondo de Adaptación (decisión 5/ CMP.27). Esta establecía que los miembros del órgano rector serían las Partes del Protocolo de Kioto, donde se regirían por un miembro un voto y donde los países No Anexo I de la CMNUCC tendrían una mayoría (Grasso, 2010:368). 


\section{Figura 1: Gobernanza del FA}

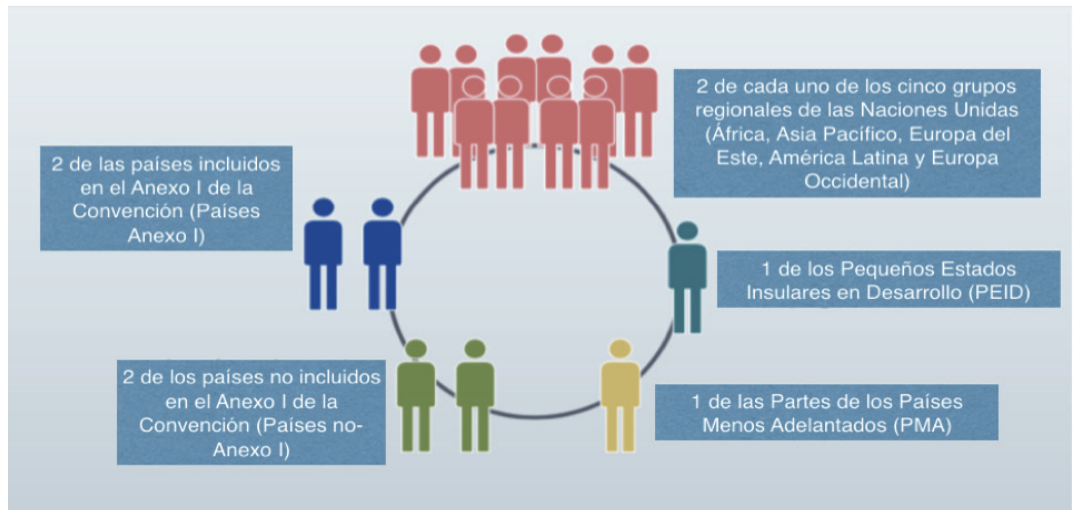

Fuente: Grimm, 2018

De esta manera, el FA es supervisado y administrado por su órgano rector, la Junta del Fondo de Adaptación (AFB). La Junta está compuesta por 16 miembros y 16 suplentes que representan a las Partes del Protocolo de Kioto (figura 1), y celebra reuniones periódicas durante todo el año (Grimm, 2018: 27). A diferencia de otros fondos, los países en desarrollo consiguieron obtener entre un 60 y $70 \%$ de representación en la Junta. Esto supone mayor voz de las comunidades más vulnerables al cambio climático, las cuales se encuentran en mayor medida en las zonas rurales de los países en desarrollo. Además, los suplentes suelen participar en las discusiones de la AFB, las cuales han sido tomadas en sus más de 10 años de funcionamiento por consenso. Además de constituir una mejora en la calidad y eficiencia en la toma de decisiones que competen al Fondo (Grimm, 2018).

La AFB toma la decisión final sobre los proyectos y la asignación de fondos (decisión 1 / CMP.3 párrafo 5; decisión 5 / CMP.2). Se distingue del resto de fondos, en que tiene la tarea específica de financiar proyectos y programas de adaptación, siendo este su objetivo final. Este órgano cuenta con un Comité de Revisión de Proyectos y Programas (PPRC), el cual los evalúa basándose en evaluaciones técnicas preparadas por la Secretaría de la AFB, quien además realiza trabajo de investigación, asesoría y administración, entre otros (Harmeling y Kaloga, 2011:5). Por su parte, el Banco Mundial actúa como fideicomisario del FA. Esto quiere decir que es quien se encarga de vender los certificados de Reducción de Emisiones de Certificados de Carbono y administra el Fondo fiduciarios del FA (Fondo de Adaptación, 2020).

En el FA es especialmente relevante el rol de la sociedad civil, pues supone un valor agregado para el Fondo debido a que está enfocado a liderar proyectos y programas orientados a las comunidades más vulnerables, donde la sociedad civil juega un rol esencial. De esta manera, el FA también supone una innovación al dar espacio a Organizaciones de la sociedad civil (OSC). Así, se creó la Red de ONG del Fondo de Adaptación (AFN), iniciada por Germanwatch en 2010 (Fondo de Adaptación, 2017:125).

No obstante, aunque la AFN participa en las discusiones sobre políticas y decisiones de financiación para la adaptación de la Junta del FA, lo hacen como observadores, sin la oportunidad de intervenir en los procedimientos. Además, las decisiones importantes son tomadas en las reuniones de los distintos comités antes de la reunión con la Junta, donde interviene la AFN. La AFN es un gran adelanto para la mejora de la transparencia del FA, con su trabajo de divulgación de recomendaciones y capacitación refuerza el rol de la sociedad civil en el FA y la comprensión pública sobre la importancia de la adaptación al cambio climático. Sin embargo, no existen canales oficiales para que la sociedad civil proporcione aportes para las reuniones, algo que supondría una verdadera revolución y democratización de la financiación climática (Grimm, 2018: 34). 


\subsection{LA MODALIDAD DE ACCESO DIRECTO DEL FONDO DE ADAPTACIÓN}

La modalidad de acceso directo es una modalidad innovadora de financiamiento climático establecida por el FA que permite a las NIEs acceder directamente al financiamiento climático y gestionar programas y proyectos de adaptación al cambio climático en todas sus etapas (Fondo de Adaptación, 2019).

Para que las NIEs puedan acceder a la modalidad de acceso directo necesitan acreditarse ante el FA. El proceso de acreditación de las NIEs es de gran relevancia para los países, ya que, debido a los rigurosos requerimientos necesarios para la acreditación, se produce un gran refuerzo institucional de la organización. Este procedimiento es válido por un periodo de cinco años. Una vez superan este tiempo, pueden volver a re-acreditarse y deben comenzar el proceso al menos nueve meses antes de finalizar el primer periodo de acreditación.

La modalidad de acceso directo del FA fue la primera en estar en pleno funcionamiento entre los fondos del clima, convirtiéndose en una modalidad pionera y distintiva del FA. Esta modalidad refuerza la capacidad de adaptación al cambio climático de los países y aprovecha la experiencia local, ya que son las entidades nacionales las que lideran la gestión de los programas y proyectos. Esto mejora el enfoque en comunidades vulnerables establecido por el FA, ya que las NIEs tienen más conocimiento sobre el contexto de las comunidades donde son implementados los programas y proyectos (Fundecooperación, 2020).

No obstante, un estudio realizado por Colenbrander et al (2017) encuentra que solo el $36,2 \%$ de los recursos comprometidos por el FA son para NIEs, el resto ha sido o será distribuido a través de Entidades Internacionales de Implementación o bancos multilaterales de desarrollo como agencias de NNUU. Además, solo un pequeño número de entidades nacionales exitosas han sido organizaciones locales de la sociedad civil, mientras que ningún gobierno sub-nacional ha recibido financiación directa de este canal. Esto se debe a distintas barreras que encuentran los actores locales para el acceso a la financiación climática. Algunas de ellas pueden ser la falta de capacidad legal, administrativa y técnica de las organizaciones para implementar proyectos de adaptación, la dificultad para incorporar la Política Social y Ambiental y la Política de Género que establece el FA, o también, los propios requerimientos económicos que hace que los donantes prefieran financiar proyectos de mayor escala ante los costos de transacción más reducidos (Colenbrander et al, 2017).

Las organizaciones locales, constituyen actores relevantes para la canalización de la financiación a las comunidades locales. Sin embargo, estas no pueden desmantelar muchas de las barreras mencionadas por sí solas. El FA desde el año 2014 ofrece hasta un monto de hasta 1 millón de dólares para que las organizaciones nacionales puedan hacer frente a dichos desafíos, además de otros instrumentos como el Programa de Preparación para el Financiamiento Climático donde ofrece apoyo a través de seminarios sobre financiamiento climático y herramientas en línea (Fondo de Adaptación, 2020). Aunque estas ayudas están dirigidas principalmente a las NIEs, estas a su vez pueden destinar ese dinero para ayudar a las organizaciones locales que participan en los proyectos. En 2015 se habilitó la modalidad de acreditación simplificada, que trata de facilitar la acreditación a aquellas organizaciones que no cuentan con todas las capacidades que se requiere para el proceso tradicional. El proceso se alinea aún más con lo demandado por el Acuerdo de París, el cual señala específicamente la necesidad de realizar procedimientos simplificados y mayor apoyo a los países en desarrollo para la preparación de capacidades (Fondo de Adaptación, 2017). 


\section{CASO DE ESTUDIO: PROGRAMA ADAPTA2+ DE FUNDECOOPERACIÓN PARA EL DESARROLLO SOSTENIBLE}

A continuación se va analizar cómo Fundecooperación realizó el proceso de canalización de financiación climática hacia dichas comunidades locales más vulnerables de Costa Rica con el programa Adapta2+. Para ello, se verá cómo las características del FA se reflejan en el caso concreto del programa.

En 2012, Fundecooperación` lograba acreditarse ante el Fondo de Adaptación como Entidad Implementadora Nacional. Esto suponía que podía hacer uso de la modalidad de acceso directo para acceder a una financiación de hasta 10 millones de dólares para proyectos orientados a mejorar la adaptación al cambio climático de las comunidades más vulnerables del país. Así, el programa Adapta2+ fue aprobado en 2014 y recibió 9.970.000 millones de euros. El programa contaba con un total de 31 proyectos divididos en toda la geografía costarricense en función de las comunidades más vulnerables identificadas (Fundecooperación, 2014).

Como se señaló en el apartado anterior, los gobiernos locales presentan muchas barreras para que la financiación climática llegue finalmente a las comunidades locales (Colenbrander, 2017). En el caso concreto de Fundecooperación, tales barreras no se encontraron debido a que el propio diseño del programa Adapta2+ consideraba abiertamente la necesidad de que el programa estuviera fundamentado en la participación de organizaciones de base y comunidades locales. No obstante, sí encontraron barreras en el proceso de acreditación ante el FA. El principal obstáculo encontrado por Fundecooperación fue referente a su capacidad administrativa y económica. Fundecooperación no tenía la capacidad suficiente como para hacer frente a distintos requisitos requeridos por el FA. Estos requisitos necesarios para lograr la acreditación entran en conflicto con el objetivo de permitir la acreditación de organizaciones locales de pequeño-mediano tamaño ante el fondo (Reyes, 2020). Más aún, el FA requería que dichos criterios necesarios para la acreditación de las NIEs también fueran cumplidos por las organizaciones locales que iban actuar como Entidad Ejecutora (EE), lo que se volvía aún más complicado, ya que eran organizaciones muy pequeñas que no contaban con casi ninguna de estas capacidades.

En lo referente a la gobernanza, para la aprobación del monto, el programa Adapta2+ tuvo que incorporar el enfoque en la comunidad que establecía el FA como requisito, el enfoque bottom-up. Así, se siguió este enfoque a través de una convocatoria abierta para que las organizaciones presentarán los distintos proyectos. Esto suponía algo inusual, puesto que la mayoría de proyectos que se habían presentado ante el FA contenían un solo EE. Los EE estaban constituidos por las organizaciones que ejecutarían las proyectos en las comunidades seleccionadas. En la mayoría de los casos, se trataba de pequeñas organizaciones que presentaron sus propuestas ante Fundecooperación para ejecutar sus proyectos en sus comunidades. Fundecooperación recibió cerca de 100 solicitudes provenientes de organizaciones públicas, privadas, locales y nacionales. Se permitió el acceso dentro de dos modalidades: un mínimo de proyectos de 50 mil dólares y un máximo de 250 mil dólares (Fundecooperación, 2018). Uno de los principales desafíos con los que contó Adapta2+ a la hora de recibir las solicitudes se debía a que muchas de las organizaciones que presentaban las propuestas no conocían la adaptación al cambio climático. Por lo que uno de los grandes retos fue dar a conocer este proceso dentro de los niveles locales y hacer ver que en muchos de estas comunidades se estaba produciendo esta resiliencia climática (Reyes, 2020).

\footnotetext{
${ }^{7}$ Fundecooperación para el Desarrollo Sostenible es una organización dedicada a impulsar la mejora de las condiciones económicas, técnico, sociales y ambientales de la población costarricense. Proporciona financiamiento y asistencia técnica tanto a los gobiernos locales como a los productores en Costa Rica (Fundecooperación, 2019).
} 
Además, Fundecooperación para asegurarse que los niveles locales estuvieran incorporados en las propuestas, estableció que si no era una organización local la que presentaba la propuesta debía estar respaldado por alguna organización de base o de las personas receptoras del proyecto. Los EE debían contar con una carta de respaldo de las organizaciones de base apoyando el proceso y, también, justificando que el proyecto que presentaban era del interés de las personas receptoras (Fundecooperación, 2018). Lo que se buscaba con este planteamiento del programa era garantizar que las propuestas fueran realizadas de manera local, de forma que las organizaciones de base pudieran presentar propuestas. No obstante, también se permitió que propuestas nacionales también pudieran participar, y es por ello que cuentan con una Secretaría Técnica Nacional y Ambiental (SETENA) o el Instituto Meteorológico de Costa Rica (IMN) (Reyes, 2020).

Estas solicitudes se sometieron a una evaluación conjunta de expertos y expertas y otras organizaciones. Actualmente, cuentan con un total de 31 proyectos y más de 80 organizaciones de base participan en ellos. Cada propuesta cuenta con un EE, pero en cada uno de ellas hay entre dos y tres organizaciones de base participando en el proceso (Fundecooperación, 2018). En el programa participan alrededor de cincuenta ASADAS, las cuales constituyen un importante indicador de que las comunidades locales están presentes en el proceso debido a su propia composición, pues se trata de órganos locales constituidos como asociaciones por delegación del Institución costarricense de Acueductos y Alcantarillados (AYA) (Monge, et al., 2013:7). Esto aseguraba que las necesidades de la comunidad donde se va implementar el proyecto estén reflejadas en las propuestas que finalmente recibirían la financiación.

\section{CONCLUSIÓN}

El FA cuenta con una serie de peculiaridades frente al resto de fondos de financiación climática que facilitan la canalización de la financiación hacia las comunidades locales. Su propia gobernanza favorece la mayor participación en la toma de decisiones de los países en desarrollo. Estos, al tener mayor participación y representación en el fondo, dan mayor visibilidad a las necesidades de las comunidades locales. Al mismo tiempo, los propios requisitos establecidos por el Fondo para financiar los distintos programas y proyectos indican la necesidad de la participación de las comunidades y organizaciones locales en los procesos. Esto asegura la intervención de los niveles locales en los programas y proyectos de una manera activa.

Por otro lado, la modalidad de acceso directo, otra de las características del FA, posibilita la mayor canalización defondos hacia las comunidades. Esto permite, através del proceso de acreditacióndelas NIEs, el manejode los recursos financieros de manera directa por parte de los países. Tal hecho ha supuesto una mejora en el acceso de las comunidades locales al Fondo. Esto ocurre puesto que ha establecido como requisito que los países que acceden a través de esta modalidad al FA canalicen los fondos hacia sus comunidades más vulnerables al cambio climático. Las NIEs son mejores conocedoras de las distintas realidades de las comunidades locales, lo que favorece la mejor adecuación de los proyectos y programas a dichas necesidades. Sin embargo, hay que tener en cuenta que muchos estudios han señalado que el simple hecho de hacer uso de la modalidad de acceso directo no asegura que las comunidades locales reciban la financiación (Colenbrander et al, 2017). Por ello, es necesario que en el diagnóstico de los países que se presentan al acceso a dicha modalidad, se recojan estas barreras. Es decir, la limitación que sufren por su propias capacidades administrativas y económicas deben ser consideradas por el propio FA y por los países. Asimismo, el FA además de realizar subvenciones para ayudar a afrontar dificultades en el proceso de acreditación, debería realizar inversiones junto con los gobiernos nacionales para mejorar las barreras nacionales que obstaculizan que las NIEs finalmente canalicen la financiación climática hacia las comunidades locales. 
Aunque se puede señalar que las comunidades locales se han visto beneficiadas por este tipo de modalidad de acceso directo del FA, se necesita repensar el conjunto de la arquitectura climática para que se reinvierta el $10 \%$ de desarrollo financiero que actualmente alcanza a los actores locales (Mostafa, et al. 2016). El FA solo es un minúsculo esfuerzo, ya que es necesario una restructuración en el conjunto de los fondos climáticos para que consigan ser accesibles a las comunidades rurales y por ende a las comunidades más vulnerables. Se requieren cambios más profundos en el conjunto de la gobernanza climática para que exista un mayor acceso y protagonismo de la sociedad civil, y más en concreto de las comunidades locales, generalmente más vulnerables al cambio climático, pues, como se señalaba en apartados anteriores, el rol de la sociedad civil en el Fondo es aún limitado. También, debe incorporar indicadores expresos referentes a los niveles locales, que no reflejen solamente el número de receptores de los proyectos, sino que se monitoree que realmente constituyen beneficiarios y existe impactos positivos en la implementación de dichos programas y proyectos. Además de que las políticas de los gobiernos locales deben estar alineadas en los programas de cambio climático para que se reflejen las necesidades y prioridades identificadas por las propias comunidades. El FA ha comenzado ese proceso de transformación, adquiriendo un rol cada vez más relevante en el conjunto de la arquitectura climática como potenciador de cambios. Sin embargo, aún se necesitan muchos cambios por hacer.

Ante la coyuntura actual, la lucha por frenar el cambio climático se ha posicionado como un elemento primordial para conservar la salud mundial. Los nuevos planteamientos hacia la oportunidad de crear un modelo económico sostenible, pasa por trabajar en realizar políticas de adaptación que creen resiliencia en las comunidades más vulnerables al cambio climático, desarrollando capacidades que les facilite afrontar el nuevo contexto internacional. Aún, con la evidencia de la relación entre la pandemia por COVID-19 y el cambio climático, muchos son los intereses de primar una recuperación económica rápida que no cuestione la estructura actual y no repiense las estructuras que sustentan la gobernanza climática, entre otras. Pero se necesita de organismos que articulen gobernanzas más democráticas donde se incorpore la voz de las comunidades locales y más vulnerables a esta coyuntura. La futura arquitectura internacional del financiamiento climático debe proporcionar mayor complementariedad entre los fondos y garantizar el uso efectivo de los recursos con procedimientos más simples y efectivos. 


\section{REFERENCIAS BIBLIOGRÁFICAS}

- Adger, X. et at. (2008) "Are there social limits to adaptation to Climate Change?". Climate Change, Vol. 93 (3), pp. 335-354. Disponible en: https://link.springer.com/article/10.1007/s10584-008-9520-z [Consulta: 15 marzo 2020]

- Cabral, R.By Bowling (2014) “Fuentes de financiamiento para el cambio climático". División de Financiamiento para el Desarrollo, CEPAL, Vol. 254, n.d. Disponible en: https://www.cepal.org/es/publicaciones/37217fuentes-financiamiento-cambio-climatico [Consulta: 20 marzo 2020]

- CEPAL (2019) Cambio Climático y Derechos Humanos. Contribuciones desde y para América Latina y el Caribe. Disponible en: https://repositorio.cepal.org/bitstream/handle/11362/44970/4/S1901157_es.pdf [Consulta: 20 marzo 2020]

- Ciplet, D., et al. (2013) "The Politics of International Climate Adaptation Funding: Justice and Divisions in the Greenhouse". Global Environmental Politics Vol. 13 (1), pp.49-68. Disponible en: https://www.researchgate.net/publication/265841882_The_Politics_of_International_Cl imate_Adaptation_Funding_Justice_and_Divisions_in_the_Greenhouse [Consulta: 1 abril 2020]

- Colenbrander, S, et alt. (2017) "Using climate finance to advance climate justice: the politics and practice of channelling resources to the local level”. Climate Policy Vol. 18 (7), pp.1-14. Disponible en: https://www. researchgate.net/publication/321637349_Using_climate_finance_to_advan ce_climate_justice_the_politics_and_practice_of_channelling_resources_to_the_local_l evel\#pf8 [Consulta: 1 abril 2020]

- Convención Marco de Naciones Unidas sobre el Cambio Climático (CMNUCC) (2020a) ¿Qué significa adaptación al cambio climático y resiliencia al clima? Disponible en: https://unfccc.int/es/topics/adaptation-and-resilience/the-big-picture/que-significa- adaptacion-al-cambio-climatico-y-resiliencia-al-clima [Consulta: 1 abril 2020]

- Convención Marco de Naciones Unidas sobre el Cambio Climático (CMNUCC) (2020b) Introducción a la mitigación. Disponible en: https://unfccc.int/es/topics/mitigation/the- big-picture/introduccion-a-la-mitigacion [Consulta: 1 abril 2020]

- Fenton, A., et al. (2014) "Up-scaling finance for community-based adaptation". Climate and Development Vol. 6, pp. 388-397. Disponible en: https://doi.org/10.1080/ 17565529.2014.953902 [Consulta: 20 marzo 2020]

- Fernández, F. (2007) "Impactos del cambio climático en las áreas urbanas y rurales" Boletín del Instituto Libre de Enseñanza. no 66-67. Disponible en: https://www.divulgameteo.es/fotos/lecturas/Impactos-CCurbanas-rurales.pdf [Consulta: 1 abril 2020]

- Fundecooperación para el Desarrollo Sostenible (2014) Full Proposal of Adata2+. Fondo de Adaptación. Disponible en: https://www.adaptation-fund.org/wpcontent/ uploads/2014/11/58CostaRicaProgramm eFullProposal-1.pdf [Consulta: 17 abril 2020] 
- Fundecooperación para el Desarrollo Sostenible (2018) Multidimensional Approach of the Adapta2+ Programme and its Relationship to: Costa Rica's Intended Nationally- Determined Contributions, Sustainable Development Goals, National Adaptation Plan and National Development Plan. Fondo de Adaptación. Disponible en: https://www.adaptationfund.org/wpcontent/uploads/2014/11/Adapta2CostaRicamultidimensional-approach-May2018.pdf [Consulta: 17 abril 2020]

- Fundecooperación para el Desarrollo Sostenible (2020) Adaptation to Coastal Erosion in Vulnerable Áreas. Disponible en: https://www.adaptationfund.org/project/adaptationto-coastal-erosion-in-vulnerable-areas/ [Consulta: 17 abril 2020]

- Fondo de Adaptación (2019) Direct Access. Disponible en: https://www.adaptation-fund.org/wp- content/ uploads/2018/11/Direct-Access-Nov-2019.pdf [Consulta: 1 abril 2020]

- Fondo de Adaptación (2020) Accreditation Apllication Disponible en: https://www.adaptation- fund.org/ apply-funding/accreditation/ [Consulta: 1 abril 2020]

- Grasso, M. (2010) "The role of justice in the North-South conflicto in climate change: the case of negotiations on the Adaptation Fund". International Enviromental Agreement, Vol. 11(4), pp.361-377. Disponible en: https://link.springer.com/article/10.1007/s10784-010- 9145-3 [Consulta: 1 abril 2020]

- Grimm, J., et al. (2018) "The future role of the Adaptation Fund in the international climate finance architecture". Germanwatch. Disponible en: http://germanwatch.org/en/15936 [Consulta: 1 abril 2020]

- Grupo Intergubernamental de Expertos sobre el Cambio Climático (IPCC) (2007) IV Informe de Cambio climático. Disponible en: https://www.ipcc.ch/site/assets/uploads/2018/02/ar4_syr_sp.pdf [Consulta: 20 marzo 2020]

- Grupo Intergubernamental de Expertos sobre el Cambio Climático (IPCC) (2014) Cambio Climático 2014. Informe de Síntesis. Disponible en: https://www.ipcc.ch/site/assets/uploads/2018/02/SYR_AR5_FINAL_ full_es.pdf [Consulta: 20 marzo 2020]

- Gutiérrez, M. (2019) “COP 25: Agujeros en la capa del financiamiento climático" El Diario, 7 de diciembre de 2019. Disponible en: https://www.eldiario.es/tribunaabierta/COP25- agujeros-capa-financiamientoclimatico_6_971212871.html [Consulta: 20 marzo 2020]

- Harmeling, S. y Kaloga, A.O. (2011) "Understanding the Political Economy of the Adaptation Fund". Institute of Deelopment Sutidies, Vol. 42 (3), n.d. Disponible en: https://opendocs.ids.ac.uk/opendocs/bitstream/ handle/20.500.12413/7604/IDSB_42_3_10.1111-j.1759- 5436.2011.00219.x.pdf;jsessionid=03F1455 024B42EFD25C49B5BFOBCEOE7?sequen ce $=1$ [Consulta: 1 abril 2020]

- Ibárcena, M. y Scheelje, JM. (2003). "Cambio climático principales causantes, secuencias y compromisos de los países involucrados". FAO. Disponible en: http://www.fao.org/3/XII/0523-B2.htm [Consulta: 20 marzo 2020]

- Khan, M. et al. (2013) "Twenty-five years of adaptation finance through a climate justice lens". International Centre for Climate Change and Development (ICCCAD). Disponible en: https://link.springer.com/article/10.1007/s10584-019-02563-x [Consulta: 20 marzo 2020] 
- Lago, R. y Urkidi, L. (n.d) "Deuda Ecológica”. Diccionario crítico de empresas transnacionales. Observatorio de multinacionales en América Latina. Asociación Paz con dignidad. Disponible en: http://omal.info/spip. php?article4833 [Consulta: 17 abril 2020]

- Lampis, A. (2013) "Vulnerabilidad y adaptación al cambio climático: debates acerca del concepto de vulnerabilidad y su medición". Cuadernos de Geografía: Revista Colombiana de Geografía, Vol. 22 (2), pp. 17-33. Disponible en: https://revistas.unal.edu.co/index.php/rcg/article/view/37017/43839 [Consulta: 17 abril 2020]

- Ministerio para la Transformación Ecológica y el Teto Demográfico (MITECO) (2020) “QQué es la adaptación al cambio climático" Disponible en: https://www.miteco.gob.es/es/cambio-climatico/temas/impactos-vulnerabilidad-y-adaptacion/plan-nacional-adaptacion-cambio-climatico/que_es_la_adaptacion. aspx [Consulta: 17 abril 2020]

- Mostafa, M., et al. (2016). "Climate adaptation funding: Getting the money to those who need it". Atomic Scientists, Vol. 72 (6), pp. 396-401. Disponible en: https://thebulletin.org/2016/11/climate-adaptationfunding-getting-the-money-to-those- who-need-it/ 43839 [Consulta: 17 abril 2020]

- Naciones Unidas (NNUU) (1992) Convención Marco de las Naciones Unidas sobre Cambio Climático. Disponible en: https://www.acnur.org/fileadmin/Documentos/BDL/2009/6907.pdf [Consulta: 20 marzo 2020]

- Ornsaran, M, et al (2020) "What makes internationally-financed climate change adaptation projects focus on local communities? A configurational analysis of 30 Adaptation Fund projects". Global Environmental Change, Vol. 61. Disponible en: https://research.wur.nl/en/publications/what-makes-internationally-financed-climate- change-adaptation-pro [Consulta: 1 abril 2020]

- Oxfam Intermón (2020) Definición de la Agenda de Acción de Accra (AAA). Disponible en: http://www.realidadayuda.org/glossary/agenda-de-accion-de-accra-aaa [Consulta: 1 abril 2020]

- Pardo, M. y Ortega, J. (2018) "Justicia ambiental y justicia climática: el camino lento pero sin retorno, hacia el desarrollo sostenible justo" Revista Castellano-Manchega de Ciencias Sociales no 24, pp- 83-100. Disponible en: https://dialnet.unirioja.es/servlet/articulo?codigo=6837003 [Consulta: 15 marzo 2020]

- PNUMA (2016) The adaptation gap report 2018. Disponible en: https://wedocs.unep.org/bitstream/handle/20.500.11822/27114/AGR_2018.pdf?sequenc e=3 [Consulta: 15 marzo 2020]

- Schipper, L. (2004) "Exploring Adaptation to Climate Change: a Development Perspective". Tyndall Center for Climate Research. School of Enviroment Sciences. University of East Anglia, no107, n.d. Disponible en: https://www.researchgate.net/publication/228391167_Climate_change_adaptation_and_ development_Exploring_the_linkages [Consulta: 15 marzo 2020]

- Schipper, L. (2007) “Climate Change adaptation and development: Exploring the linkages”. Tyndall Center for Climate Research. School of Enviroment Sciences. University of East Anglia, no107, n.d. Disponible en:https://www.researchgate.net/publication/228391167_Climate_change_adaptation_a nd_development_Exploring_the_linkages [Consulta: 15 marzo 2020]

- Stern, N. (2006) Informe sobre la economía del cambio climático. Cambridge University Press. Disponible en: www.sternreview.org.uk [Consulta: 20 marzo 2020] 
- Reyes, C. (2020). Entrevista A Carolina Reyes Sobre El Programa De Adapta2+. San José, Costa Rica. Realizada el 6 de marzo de 2020.

- Ruíz-Gauma, I. et al. (2020) " Financiando las políticas climáticas y de sostenibilidad: el impacto de los bonos sostenibles en el País Vasco" Ekonomiaz no 97. Disponible en: https://dialnet.unirioja.es/servlet/ articulo?codigo=7536538 [Consulta: 20 marzo 2020]

- Urrutia, S. (2010). "El régimen jurídico internacional del cambio climático después del Acuerdo de Copenhague". Revista de Derecho de la Pontifica, no 34, pp. 597-633. Universidad Católica de Valparaíso. Disponible en: https://scielo.conicyt.cl/scielo.php?script=sci_arttext\&pid=S0718-68512010000100019 [Consulta: 20 marzo 2020]

- Yamin, F., et al. (2005) "Vulnerability, Adaptation and Climate Disasters: A conceptual Overview". Institute of Development Studie, Vol. 36 (4), n.d. Disponible en: https://www.unisdr.org/files/7789_Overview3641. pdf [Consulta: 15 marzo 2020] 
